Original Contribution

\title{
Silybin combined with phosphatidylcholine and vitamin E in patients with nonalcoholic fatty liver disease: A randomized controlled trial
}

\author{
Carmela Loguercio ${ }^{\mathrm{a}, *}$, Pietro Andreone ${ }^{\mathrm{b}}$, Ciprian Brisc ${ }^{\mathrm{c}}$, Michaela Cristina Brisc ${ }^{\mathrm{c}}$, Elisabetta Bugianesi ${ }^{\mathrm{d}}$, \\ Maria Chiaramonte e , Carmela Cursaro ${ }^{b}$, Mirela Danila ${ }^{\mathrm{f}}$, Ilario de Sio ${ }^{\mathrm{a}}$, Annarosa Floreani ${ }^{\mathrm{g}}$, \\ Maria Antonietta Freni ${ }^{\mathrm{h}}$, Antonio Grieco ${ }^{\mathrm{i}}$, Marzia Groppo ${ }^{\mathrm{j}}$, Roberta Lazzari ${ }^{\mathrm{g}}$, Salvatore Lobello ${ }^{\mathrm{g}}$, \\ Elisabetta Lorefice ${ }^{\mathrm{k}}$, Marzia Margotti ${ }^{\mathrm{b}}$, Luca Miele ${ }^{\mathrm{i}}$, Stefano Milani ${ }^{\mathrm{k}}$, Lajos Okolicsanyi ${ }^{\mathrm{j}}$, \\ Giuseppe Palasciano ", Piero Portincasa ${ }^{\text {, }}$ Patrizia Saltarelli ${ }^{m}$, Antonina Smedile ${ }^{\mathrm{d}}$, Francesco Somalvico ${ }^{\mathrm{n}}$, \\ Aldo Spadaro ${ }^{\mathrm{h}}$, Ioan Sporea ${ }^{\mathrm{f}}$, Paolo Sorrentino ${ }^{\mathrm{o}}$, Raffaela Vecchione ${ }^{\mathrm{o}}$, Concetta Tuccillo ${ }^{\mathrm{a}}$, \\ Camillo Del Vecchio Blanco ${ }^{a}$, Alessandro Federico ${ }^{\text {a }}$
}

a Department F. Magrassi e A. Lanzara, Second University of Naples, 80131 Napoli, Italy

b Clinical Medicine, University of Bologna, Italy

c Gastroenterology, University of Oradea, Oradea, Romania

d Gastroenterology and Hepatology, Hospital Molinette, Torino, Italy

e Gastroenterology, Hospital Sacro Cuore Don Calabria, Negrar, VR, Italy

${ }^{\mathrm{f}}$ Gastroenterology and Hepatology, Hospital Judetean Timisoara, Timisoara, Romania

g Gastroenterology, Hospital Sant'Antonio, Padova, Italy

${ }^{\mathrm{h}}$ Medical Clinic Policlinico Martino, Messina, Italy

${ }^{\mathrm{i}}$ Internal Medicine, Catholic University Sacred Heart, Rome, Italy

j Gastroenterology, Treviso Hospital, Treviso, Italy

${ }^{\mathrm{k}}$ Gastroenterology, Hospital Careggi, Firenze, Italy

${ }^{1}$ Medical Clinic Augusto Murri, University of Bari, Bari, Italy

$\mathrm{m}$ Gastroenterology and Hepatology, University of L'Aquila, L'Aquila, Italy

n Society of Statistics, Milan, Italy

${ }^{\circ}$ Anatomical Pathology, University Federico II, Napoli, Italy

\section{A R T I C L E I N F O}

\section{Article history}

Received 29 September 2011

Revised 31 January 2012

Accepted 5 February 2012

Available online 15 February 2012

\section{Keywords:}

Nonalcoholic fatty liver

Silybin

Hepatoprotectant

Antioxidant

Free radicals

\begin{abstract}
A B S T R A C T
The only currently recommended treatment for nonalcoholic fatty liver disease (NAFLD) is lifestyle modification. Preliminary studies of silybin showed beneficial effects on liver function. Realsil (RA) comprises the silybin phytosome complex (silybin plus phosphatidylcholine) coformulated with vitamin E. We report on a multicenter, phase III, double-blind clinical trial to assess RA in patients with histologically documented NAFLD. Patients were randomized 1:1 to RA or placebo (P) orally twice daily for 12 months. Prespecified primary outcomes were improvement over time in clinical condition, normalization of liver enzyme plasma levels, and improvement of ultrasonographic liver steatosis, homeostatic model assessment (HOMA), and quality of life. Secondary outcomes were improvement in liver histologic score and/or decrease in NAFLD score without worsening of fibrosis and plasma changes in cytokines, ferritin, and liver fibrosis markers. We treated 179 patients with NAFLD; 36 were also HCV positive. Forty-one patients were prematurely withdrawn and 138 patients analyzed per protocol (69 per group). Baseline patient characteristics were generally well balanced between groups, except for steatosis, portal infiltration, and fibrosis. Adverse events (AEs) were generally transient and included diarrhea, dysgeusia, and pruritus; no serious AEs were recorded. Patients receiving RA but not $\mathrm{P}$ showed significant improvements in liver enzyme plasma levels, HOMA, and liver histology. Body mass index normalized in $15 \%$ of RA patients (2.1\% with P). HCV-positive patients in the RA but not the P group showed improvements in fibrogenesis markers. This is the first study to systematically assess silybin in NAFLD patients. Treatment with RA but not P for 12 months was associated with
\end{abstract}

Abbreviations: AE, adverse event; AST, aspartate aminotransferase; ALT, alanine aminotransferase; BMI, body mass index; HCV, hepatitis C virus; HOMA, homeostatic model as-

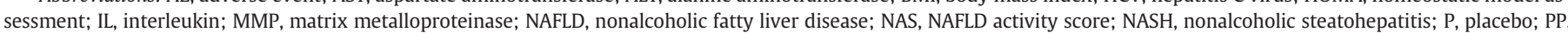
per protocol; SF-36, short form 36; TGF, transforming growth factor; TIMP, tissue inhibitor of metalloproteinase; TNF, tumor necrosis factor; $\gamma$ GT, $\gamma$-glutamyltranspeptidase.

* Corresponding author. Fax: + 39815666718.

E-mail address: carmelina.loguercio@unina2.it (C. Loguercio). 
improvement in liver enzymes, insulin resistance, and liver histology, without increases in body weight. These findings warrant further investigation.

Nonalcoholic fatty liver disease (NAFLD) is an important emerging liver damage epidemic. When characterized by only steatosis, NAFLD is associated with increased cardiovascular risk and worsens the course of hepatitis $\mathrm{C}$; when represented by nonalcoholic steatohepatitis (NASH), NAFLD can evolve into liver fibrosis and cirrhosis [1,2]. Currently there is no effective therapy for NAFLD, except for lifestyle modification [3]. Both dysregulated glucose metabolism and oxidative stress in hepatocytes are key pathogenic factors in the onset of steatosis and progression of NASH, its inflammatory and potentially evolutive form. Studies have shown that insulin-sensitizing agents and antioxidants may improve clinical and histologic features of NAFLD, although data remain inconclusive $[4,5]$.

Silybin is a potent antioxidant representing about $50-70 \%$ of the silymarin extract of Silybum marianum (milk thistle). Silybin modulates inflammation and fibrogenesis and interferes with intrahepatic glycolysis and gluconeogenesis [6,7]. Silybin treatment attenuated liver damage and diabetes in animal models of NASH and type 1 diabetes mellitus [8-11] and showed benefits in patients with poorly controlled type 2 diabetes with or without alcoholic liver cirrhosis [12,13].

As with other flavolignans, limitations of silybin use include low water solubility, low bioavailability, and poor intestinal absorption [14]. Derivatives of silybin with improved solubility may surmount these pharmacologic limitations $[15,16]$. The silybin phytosome complex (silybin plus phosphatidylcholine) has been coformulated with vitamin E (Realsil (RA); Istituto Biochimico Italiano, Lorenzini S.p.a., Italy). Pharmacokinetic analyses indicated that silybin phytosome bioavailability is much higher than silymarin bioavailability $[17,18]$.

Given the potentially beneficial effects of silybin on liver function, and the need for effective therapies for liver steatosis, we carried out a placebo-controlled, double-blind, phase III, randomized clinical trial to assess RA in NAFLD patients.

\section{Experimental procedures}

\section{Trial design}

The trial was conducted at 11 Italian and 2 Romanian centers. Protocol and patient consent forms were designed by the coordinating center and reviewed by all participating centers. The protocol conformed to ethical guidelines of the 1975 Declaration of Helsinki and was approved by the Institutional Review Board at each participating center. All patients gave informed written consent. The trial was registered with the European Clinical Trials Database (EudraCT, Ref. 2005-000860-24). No significant changes were made to the protocol or prespecified outcomes after trial commencement.

Inclusion criteria were histologically documented liver steatosis or steatohepatitis diagnosed within 12 months, age $18-65$, persistent increase in $\geq 1$ plasma aminotransferase (aspartate aminotransferase (AST) and/or alanine aminotransferase (ALT)) and/or $\gamma$ glutamyltranspeptidase ( $\gamma \mathrm{GT})$ within 6 months, and negative pregnancy test (females). Hepatitis C virus (HCV)-positive patients (confirmed by HCV RNA assay) + NAFLD with prior HCV treatment failure could enroll. Even if both pathogenesis and treatment of HCV-related chronic hepatitis were very different from NAFLD/ $\mathrm{NASH}$, in this study we aimed to verify the effect of RA on the metabolic component of fatty liver also in HCV-positive patients.

Exclusion criteria were liver cirrhosis, presence of other major diseases including type 1 diabetes, hepatitis B or D infection, genotype 3 $\mathrm{HCV}$ infection, any treatment for liver disease or HCV infection within
6 months, participation in other trials, hypersensitivity to treatment, daily ethanol consumption $\geq 20 \mathrm{~g}$, and substance abuse. Drinking habits were assessed by questionnaires on lifetime drinking history and self-administered AUDIT C test $[19,20]$. Patients with normal values of one liver enzyme at baseline $\left(T_{0}\right)$ were not excluded.

Patients were allocated (1:1) to receive active treatment (RA; active components: silybin $94 \mathrm{mg}$, phosphatidylcholine $194 \mathrm{mg}$, vitamin E acetate $50 \%$ ( $\alpha$-tocopherol $30 \mathrm{mg}$ ) $89.28 \mathrm{mg}$ ) or placebo (P; extrawhite saccharine replacing active components) orally twice daily for 12 consecutive months, on a consecutive basis within each site according to a centrally randomized list generated by block allocation. The block allocation sequence was based on numbers randomly generated by a computer program and randomization was not stratified. Investigators recorded patients' randomization numbers on the case report form. Patients and investigators were blinded to treatment until trial completion; treatment assignment code was sealed in the randomization envelopes.

Other treatments were disallowed. Patients received recommendations for lifestyle modification and an individually designed diet, plus dietary recommendations including reduction of simple carbohydrates (glucose, sucrose, fructose), fatty dressings (butter, mayonnaise), fatty cheeses, and sausages and increases in vegetable proteins and fiber.

Patients with $<70 \%$ treatment compliance or who missed a visit were withdrawn.

\section{Clinical and laboratory assessments}

Clinical history, physical examination, and liver biopsy results were recorded at baseline; histology was defined by NAFLD activity score (NAS) [21]. NAS uses a standardized grading system for steatosis (0 to 3), lobular inflammation (0 to 3 ), and hepatocellular ballooning ( 0 to 2 ), higher scores indicating increased severity. Monthly check-ups assessed compliance, adverse events (AEs), and drug delivery; patients had to record their drug consumption daily. AST, ALT, and $\gamma$ GT plasma levels were determined at baseline and every 3 months. The following assessments were completed at baseline, 6 months $\left(T_{6}\right)$, and 12 months $\left(T_{12}\right)$ : body mass index (BMI; overweight, BMI $\geq 25$ and $<30 \mathrm{~kg} / \mathrm{m}^{2}$; obese, BMI $\geq 30 \mathrm{~kg} / \mathrm{m}^{2}$ ); abdominal circumference and waist-to-hip ratio; abdominal ultrasonography (severity graded on semiquantitative scale: 0 , absent; 1 , mild; 2, moderate; and 3, severe) [22]; quality of life (QoL; 36-item short-form health survey (SF-36)) [23]; routine biochemistry tests (blood urea nitrogen, creatinine, bilirubin, total protein, alkaline phosphatase, uric acid, blood cell count, and prothrombin activity); HCV RNA levels (HCV-positive patients); plasma levels of glucose, triglycerides, cholesterol, ferritin, and insulin; and homeostatic model assessment (HOMA) [24]. Cytokines (tumor necrosis factor $\alpha$ (TNF- $\alpha$ ), interleukin 10 (IL-10), transforming growth factor $\beta$ (TGF- $\beta$ )) and liver fibrosis markers (hyaluronic acid, matrix metalloproteinase 2 (MMP-2), procollagens I and III, tissue inhibitor of metalloproteinases 1 and 2 (TIMP-1 and -2)) were assessed at baseline and at $T_{12}[25,26]$. A second liver biopsy was performed at $T_{12}$ if allowed by the Institutional Review Board and the patient had resigned the informed consent form.

Fasting blood samples were collected and plasma for cytokine level assessment was stored at $-80^{\circ} \mathrm{C}$. Cytokines and markers of liver fibrosis were assessed by the coordinating center using enzyme-linked immunosorbent assay kits (IL-10, TNF- $\alpha$, MMP-2, TGF- $\beta$, R\&D Systems, Minneapolis, MN, USA; hyaluronic acid, Echelon 
Biosciences, Salt Lake City, UT, USA; procollagen I, Takara Bio, Otsu, Shiga, Japan; procollagen III, USCN Life Science, Wuhan, China; TIMP-1, Invitrogen, Carlsbad, CA, USA; TIMP-2, Chemicon International, Millipore, Billerica, MA, USA).

Although trial eligibility was determined by each site's pathologist using locally prepared tissue sections, the final analysis used centrally prepared sections (stained with hematoxylin and eosin, Chromotrope aniline blue trichrome, Sweet's reticulin, Sirius red, periodic acidSchiff, periodic acid-Schiff diastase, and Pearl's and immunostained for ubiquitin) reviewed by two pathologists (blinded to clinical data/treatment) with liver histopathology experience. Steatohepatitis was assessed using a protocol incorporating histologic features for NASH grading/staging [21,27] and using NAS. Liver damage (HCVinfected patients) was graded by Ishak score [28], with evaluation also of perisinusoidal/pericellular fibrosis when present.

\section{Statistical analyses}

Sample size assumptions incorporated preliminary data [29,30], showing $23-25 \%$ efficacy with RA versus $6-10 \%$ with P. Additional assumptions included improvement in clinical condition, normalization of biochemical index, planned accrual period of 1 year, and minimum 6 -month treatment period. Estimated sample size (two-tailed $\alpha$ error of $5 \%, \beta$ potency of $90 \%$ ) was 90 patients per group plus an estimated lost-to-follow up rate of $10 \%$ (200 patients in total).

Prespecified primary outcomes were clinical conditions; AST, ALT, and/or $\gamma$ GT plasma level normalization; ultrasonographic liver steatosis improvement; HOMA; and QoL (SF-36). Secondary outcomes were liver histologic score improvement of hepatocellular steatosis points and/or NAFLD activity score decrease points, without fibrosis worsening, and plasma level changes in cytokines, ferritin, and liver fibrosis markers.

Based on residual treatment sachets presented at check-ups, patient compliance was calculated as $\left(1-\left(V_{\mathrm{a}}-V_{\mathrm{o}}\right) / V_{\mathrm{a}}\right) \times 100\left(V_{\mathrm{a}}\right.$ is the number of sachets a patient received; $V_{0}$ is the number of sachets returned).

The efficacy evaluation of the study made with the intention to treat analysis and with the per protocol analysis was aimed at estimating the frequency of success at the final visit (for the subjects considered responders). An interim analysis was done at the 6th month. According to protocol, all data were described with contingency tables and with classical descriptive statistics (minimum, maximum, media, standard deviation, median, etc). The entire hypothesis was tested with a double-tailed test.

Primary and secondary outcomes are reported separately for HCVnegative and -positive patients. All analyses are per protocol (PP), except liver histology $\left(T_{0}\right.$ versus $\left.T_{12}\right)$ analyzed in all randomized patients who received treatment $\geq 6$ months with compliance $\geq 70 \%$. PP analyses excluded patients not meeting inclusion/exclusion criteria, consuming disallowed medication, with compliance $<70 \%$, or not attending the final visit. Data missing after $T_{12}$ were imputed by last observed carried forward. No exploratory or post hoc analyses were conducted.

The statistical tests used to evaluate the efficacy and tolerability of treatments were done with a significance level of $5 \%(p<0.05)$ to ensure an error of $5 \%$.

The evaluation of the study was planned with intention-to-treat analysis, considering all the primary variables and all the secondary variables. Also a PP analysis was planned, considering the primary variables and the analysis of the histological scores.

\section{Primary end-point analysis}

The primary efficacy variable was to be analyzed using doubleentry contingent tables and $\chi^{2}$ test. The primary variables between and into the groups at any time ( $0,3,6,9$, and 12 months) were to be evaluated using the analysis of variance, whereas the nonparametric data were evaluated by means of McNemar or Cochran test and $\chi^{2}$ test.

An eventual analysis could be made to quantify the association between two or more variables with correlation test or multivariate analysis.

\section{Secondary end-point analysis}

The evaluation of secondary variables was planned for dichotomic variables with nonparametric tests, whereas for continuous variables, parametric or nonparametric tests were used, according to sample distribution. The statistical evaluation of data was performed for any time of the study $(0,3,6,9$, and 12 months).

Demographic data were analyzed by descriptive statistics including mean, standard deviation, standard error of the mean (SE) and 95\% confidence interval. Sample homogeneity for descriptive variables was verified by $\chi^{2}$ test or Fisher exact test; continuous variables were verified by Student $t$ test (independent data). Normal distribution of data was assessed by Kolmogorov-Smirnov test. For continuous variables, differences between or within groups was analyzed by two-tailed analysis of variance. Tukey-Friedman and Wilcoxon tests were used for multiple comparisons. The Mann-Whitney test was used to analyze data between groups if the data were not normally distributed. Discrete/codified variables and dichotomic variables were analyzed over time by Cochran or McNemar test and between groups by $\chi^{2}$ test. The Cohen $\kappa$ test was performed to verify concordance of the clinical diagnosis index. Spearman's rank correlation index $(r)$ was used to investigate the association between clinical and biochemical data and liver histology.

Statistical analyses were performed using SPSS (version 17; IBM Corp., Somers, NY, USA) and/or Statistica (version 8.0; StatSoft, Tulsa, OK, USA) software. nQuery Advisor (version 4.0; Statistical Solutions Ltd, Cork, Ireland) was used for sample size calculation.

\section{Results}

The trial took place from 13 June 2005 to 10 October 2008 and enrolled 180 patients with histologically proven NAFLD; 36 were HCV positive. Overall, 91 patients received RA and 88 received P. Fortyone patients were prematurely withdrawn (22 and 19 from the RA and P groups, respectively), with 138 patients available for PP analysis (69 in each group). Reasons for discontinuation were physician's decision $(n=5)$, patient's decision $(n=21)$, AEs $(n=10)$, or other $(n=5)$. AEs were generally transient and included diarrhea, dysgeusia, and abdominal pain. No serious AEs related to the study drug were recorded.

Baseline characteristics were generally well balanced between groups. One hundred eight patients entered the study with NAFLD (RA group, M/F 47/8, mean age $40.8 \pm 10.3$, BMI $29.9 \pm 4.6$, waist/ hip ratio $0.94 \pm 0.09$, abdominal circumference $105 \pm 10 \mathrm{~cm}$; P group, M/F 43/10, mean age $44.2 \pm 9.5$, BMI $29.3 \pm 4.4$, waist/hip ratio $0.95 \pm 0.09$, abdominal circumference $102 \pm 12 \mathrm{~cm}$ ) and $30 \mathrm{pa}-$ tients with NAFLD and HCV chronic infection (RA group, M/F 5/9, mean age $46.9 \pm 10.2$, BMI $27.6 \pm 3.6$, waist/hip ratio $0.89 \pm 0.08$, abdominal circumference $103 \pm 12 \mathrm{~cm}$; P group, M/F 7/9, mean age $50.9 \pm 9.5$, BMI $27.8 \pm 3.4$, waist/hip ratio $0.91 \pm 0.06$, abdominal circumference $102 \pm 15 \mathrm{~cm}$ ). The main biochemical findings are reported in Table 1. Basal hypercholesterolemia was found in about $50 \%$ of subjects in all groups, hypertriglyceridemia in a range from 20 to $49 \%$, and hyperferritinemia in $20-30 \%$ of cases, without significant differences in all groups. Under basal condition, both cytokines and plasma markers of liver fibrosis did not differ from normal values or among groups. The score for ultrasonographic liver steatosis was 1 or 2 in about $40 \%$ of cases each, whereas it was 3 in only $20 \%$ of patients. No differences for this parameter were registered among the groups. 
Table 1

Baseline main biochemical findings of patients evaluated by PP analysis.

\begin{tabular}{|c|c|c|c|c|c|c|}
\hline & \multicolumn{3}{|c|}{ NAFLD $(n=108)$} & \multicolumn{3}{|c|}{$\begin{array}{l}\text { NAFLD and HCV infection } \\
(n=30)\end{array}$} \\
\hline & RA group & P group & $\begin{array}{l}p \\
\text { (RA vs P) }\end{array}$ & RA group & P group & $\begin{array}{l}p \\
(\text { RA vs P) }\end{array}$ \\
\hline HOMA & $4.9 \pm 3.6$ & $4.6 \pm 3.0$ & n.s. & $6.3 \pm 4.1$ & $4.8 \pm 3.3$ & n.s. \\
\hline AST (IU/L) & $52 \pm 40$ & $56 \pm 20$ & n.s. & $75 \pm 30$ & $83 \pm 70$ & n.s. \\
\hline ALT (IU/L) & $49 \pm 35$ & $52 \pm 30$ & n.s. & $84 \pm 40$ & $87 \pm 32$ & n.s. \\
\hline$\gamma \mathrm{GT}(\mathrm{IU} / \mathrm{L})$ & $73 \pm 30$ & $75 \pm 31$ & n.s. & $74 \pm 40$ & $79 \pm 23$ & n.s. \\
\hline $\begin{array}{c}\text { Cholesterol } \\
(\mathrm{mg} / \mathrm{dl})\end{array}$ & $200 \pm 50$ & $190 \pm 35$ & n.s. & $197 \pm 40$ & $189 \pm 30$ & n.s. \\
\hline $\begin{array}{l}\text { Triglycerides } \\
(\mathrm{mg} / \mathrm{dl})\end{array}$ & $153 \pm 65$ & $160 \pm 58$ & n.s. & $154 \pm 45$ & $167 \pm 55$ & n.s. \\
\hline $\begin{array}{l}\text { Ferritin } \\
\quad(\mathrm{ng} / \mathrm{ml})\end{array}$ & $220 \pm 100$ & $234 \pm 95$ & n.s. & $240 \pm 45$ & $237 \pm 70$ & n.s. \\
\hline
\end{tabular}

$\overline{\text { Means } \pm \text { standard deviation. Total } n=138 \text {. PP, per protocol; NAFLD, nonalcoholic fatty }}$ liver disease; RA, Realsil; P, placebo; HOMA, homeostatic model assessment; AST, aspartate aminotransferase; ALT, alanine aminotransferase; $\gamma \mathrm{GT}, \gamma$-glutamyltranspeptidase; IU, international units; n.s., not significant.

\section{Primary outcomes in NAFLD patients}

\section{Liver enzymes}

Baseline plasma values of AST, ALT, or $\gamma \mathrm{GT}$ were in the normal range in many patients. Fig. 1 summarizes the liver enzyme changes. With RA, but not $\mathrm{P}$, the proportion of patients with normalized AST levels progressively increased ( 43 and $15 \%$ in the RA and P groups, respectively, $p<0.001$ at $T_{12}$ in the RA group vs baseline). Similarly, the proportion of patients with normalized ALT values significantly increased over time in the RA but not in the P group ( $41 \%$ vs $5 \%$, respectively at $T_{12} ; p<0.05, p<0.001$, and $p<0.05$ vs baseline at $T_{3}, T_{9}$, and $T_{12}$, respectively). No significant differences were observed between $\mathrm{RA}$ and $\mathrm{P}$ at $T_{12}$.

A significant improvement was observed in $\gamma \mathrm{GT}$ with RA ( $p=0.0002$ at $T_{12}$ vs baseline) but not with P. Accordingly, the proportion of patients with normalization of $\gamma \mathrm{GT}$ levels was higher with RA than with $\mathrm{P}$ from $T_{3}$ onward (45\% vs $19 \%$, respectively, at $T_{3}, p<0.05$ vs baseline; $p<0.001$ vs baseline at $T_{6}, T_{9}$, and $T_{12}$ ). For $\gamma \mathrm{GT}$, a significant difference was also found between groups $\left(p=0.03\right.$ at $\left.T_{12}\right)$. Of patients with elevated baseline AST, ALT, or $\gamma$ GT (Fig. 2), significantly more reached normal levels with RA versus $P$ at $T_{12}(p<0.01$ for each enzyme).

\section{Ultrasonographic liver steatosis}

The degree of liver steatosis, assessed by ultrasonography, was not statistically different between RA and $\mathrm{P}$ at $T_{12}$. In patients with moderate or severe steatosis (score 2 or 3 ), improvements tended to be greater at $T_{12}$ among those receiving RA versus P (41 and $29 \%$, respectively; not significant).

BMI

At baseline, 10 patients receiving RA and 10 receiving placebo had normal BMI. In the RA and $P$ groups, respectively, 33 and 38 patients were overweight and 26 and 21 patients were obese. Adherence to individualized diets was poor in all patients; at $T_{12}$ no significant changes in mean BMI were observed in any group. Waist-hip ratio and abdominal circumference were unaffected by treatment. Among overweight and obese patients (approximately 85\%), BMI had normalized at $T_{12}$ in $15 \%$ of RA patients ( $p<0.01$ vs baseline) versus $2.1 \%$ of $P$ patients (not significant). No patient's BMI worsened during the study, and no relationship was found among BMI, liver enzyme, and HOMA changes.

HOMA

At baseline four patients had diabetes, one of whom was HCV positive. At $T_{12}$, blood glucose was $31 \%$ lower versus baseline in RA-
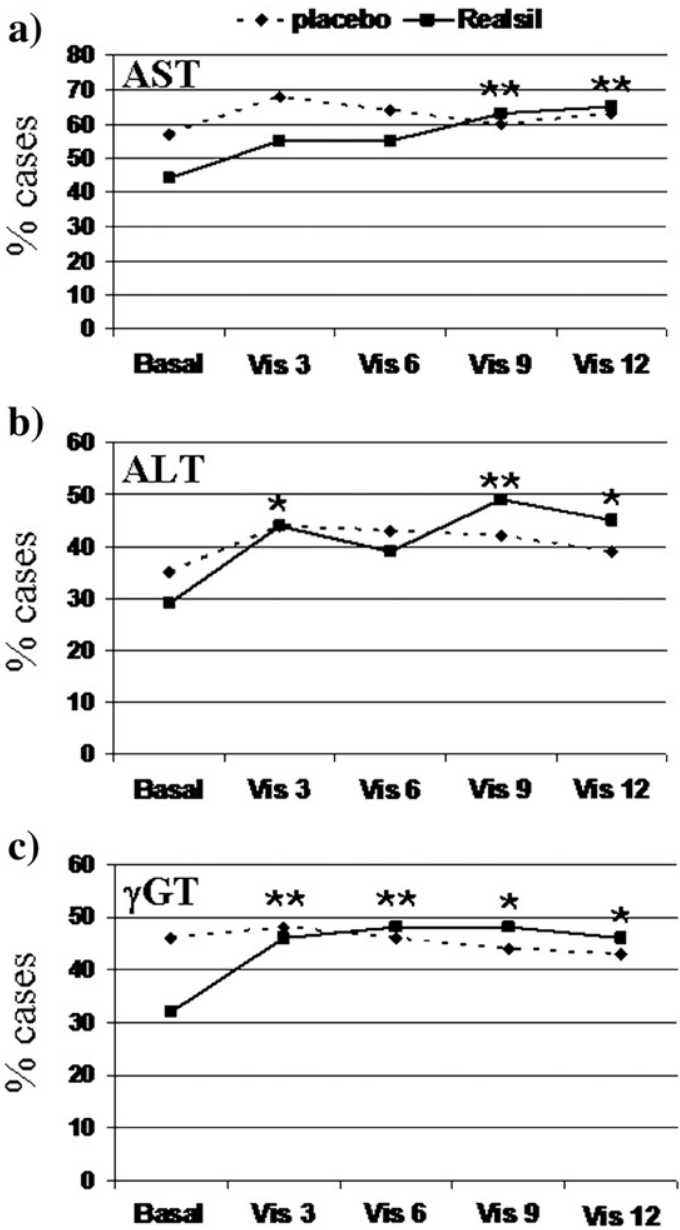

Fig. 1. Proportions of patients whose plasma levels of (a) AST, (b) ALT, and (c) $\gamma$ GT normalized over time. $p=0.03$ for $\gamma$ GT between RA and P at $T_{12}{ }^{*} p<0.05$ vs basal; ${ }^{* *} p<0.001$ vs basal. ALT, alanine aminotransferase; AST, aspartate aminotransferase; $\gamma$ GT, $\gamma$-glutamyltranspeptidase; P, placebo; RA, Realsil; $T_{12}, 12$ months; Vis, visit at month.

treated patients, with no notable difference from baseline in the $\mathrm{P}$ group (not significant between groups). At $T_{12}$, insulinemia was significantly lower than at baseline with RA $(p=0.02)$ but not with $\mathrm{P}$ $(p=0.72)$. HOMA was significantly reduced from baseline to $T_{12}$ with RA $(>10 \%, p=0.09)$ but not with P. Among patients with a baseline HOMA > 2.7 (43 and 39 patients with RA and P, respectively; arbitrarily selected threshold), HOMA progressively decreased from $T_{6}$ onward in the RA group (Fig. 3). In the P group, HOMA transiently decreased at $T_{6}$ but returned to baseline levels by $T_{12}$.

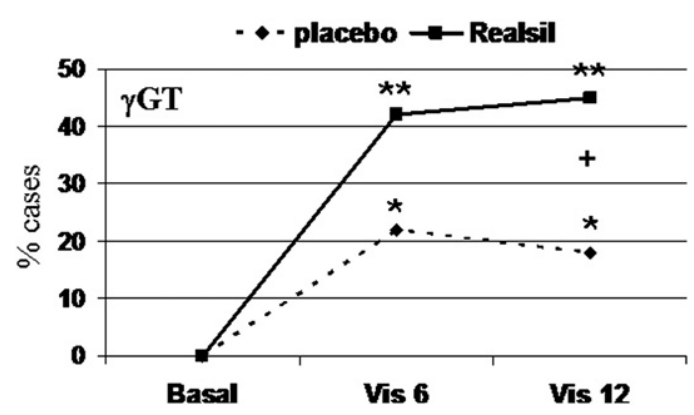

Fig. 2. Proportion of patients with normalized $\gamma \mathrm{GT}$ plasma values among those with abnormal $\gamma$ GT levels at baseline. ${ }^{*} p<0.05$ vs basal; ${ }^{* *} p<0.001$ vs basal; ${ }^{+} p<0.001$ for RA vs P group. $\gamma \mathrm{GT}, \gamma$-glutamyltranspeptidase; P, placebo; RA, Realsil; Vis, visit at month. 


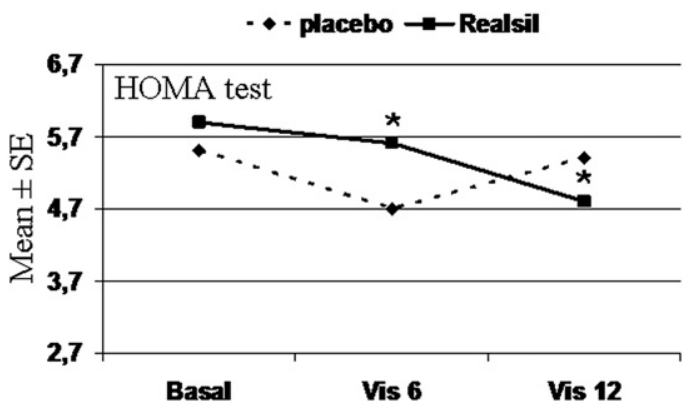

Fig. 3. Mean HOMA in patients with HOMA values $>2.7$ at basal. ${ }^{*} p<0.05$ vs basal. HOMA, homeostatic model assessment; Vis, visit at month.

QoL

In patients completing the study, treatment compliance was good. QoL did not worsen during the study and no significant changes from baseline were observed.

\section{Secondary outcomes in NAFLD patients}

At baseline, 63 patients had only liver steatosis; 45 had NASH according to established scores [21]. Among HCV-positive patients, 23 (77\%) had only steatosis and 7 had NASH. Only 35 patients agreed to liver biopsy after treatment: 32 with NAFLD ( 9 of whom had $\mathrm{NASH}$ ) and $3 \mathrm{HCV}$-positive patients (1 with NASH). Results are given for this subset of double-biopsied patients receiving 12 months' treatment.

At baseline, there were significant correlations among several clinical, biochemical, and histologic characteristics (Table 2). Histologic changes over time are summarized in Table 3. The mean severity score for liver steatosis significantly decreased from baseline to $T_{12}$ with RA ( $38 \%, p=0.004$ ) but not P. Lobular inflammation, ballooning, and NAS were significantly improved by RA; liver fibrosis was significantly reduced between baseline and $T_{12}$ with RA ( $p=0.023$ ) but not P. No significant differences were found any of the groups for other histological characteristics evaluated, such as ubiquitin, Mallory bodies, portal infiltration, and siderosis.

When looking at the NAS comparison, the end results are very similar numbers ( 3.47 vs 3.32); however, at the end of the study, after the randomization and the central revision of the histological samples, a discrepancy between RA and P groups under basal conditions was found, and the RA group started with a higher NAS with respect to that of the P group.

At baseline, TGF- $\beta$ levels were significantly inversely correlated with ballooning $(p=0.004)$ and NAS $(p=0.008)$ and directly correlated with fibrosis $(p=0.009)$. TNF- $\alpha$ levels were significantly correlated with histologic steatosis $(p<0.01)$. Alterations in plasma levels of cytokines were sporadic and not influenced by treatment.
Table 3

Effect of RA or P treatment on liver histology at $T_{12}$.

\begin{tabular}{|c|c|c|c|c|c|c|}
\hline & \multicolumn{3}{|c|}{ RA group } & \multicolumn{3}{|l|}{ P group } \\
\hline & $T_{0}$ & $T_{12}$ & $p\left(T_{0} \mathrm{vs} T_{12}\right)$ & $T_{0}$ & $T_{12}$ & $p\left(T_{0} \operatorname{vs} T_{12}\right)$ \\
\hline Steatosis & & & 0.004 & & & 0.32 \\
\hline Low/moderate & 40 & 75 & & 83 & 83 & \\
\hline Severe & 60 & 25 & & 17 & 17 & \\
\hline $\begin{array}{l}\text { Lobular } \\
\text { inflammation }\end{array}$ & & & 0.013 & & & 0.11 \\
\hline Absent/low & 63 & 89 & & 100 & 100 & \\
\hline Moderate & 37 & 11 & & 0 & 0 & \\
\hline Ballooning & & & 0.009 & & & 0.11 \\
\hline Absent/low & 50 & 90 & & 75 & 73 & \\
\hline Moderate & 50 & 30 & & 25 & 25 & \\
\hline Fibrosis & & & 0.023 & & & 0.69 \\
\hline Absent/minimal & 20 & 60 & & 75 & 66 & \\
\hline Moderate & 25 & 5 & & 0 & 17 & \\
\hline Portal-periportal & 15 & 25 & & 17 & 9 & \\
\hline Perisinusoidal & 35 & 5 & & 0 & 0 & \\
\hline Bridging fibrosis & 5 & 5 & & 8 & 8 & \\
\hline NAS $($ mean $\pm S D)$ & $\begin{array}{l}5.05 \pm \\
1.35\end{array}$ & $\begin{array}{l}3.47 \pm \\
1.95\end{array}$ & 0.003 & $\begin{array}{l}3.90 \pm \\
0.88\end{array}$ & $\begin{array}{l}3.32 \pm \\
1.93\end{array}$ & 0.13 \\
\hline
\end{tabular}

RA, Realsil; P, placebo; $T_{0}$, baseline; $T_{12}, 12$ months of treatment; NAS, nonalcoholic fatty liver disease activity score.

Plasma ferritin levels were normal, or close to normal, at baseline and throughout the study. Additional metabolic parameters, including plasma cholesterol and triglycerides, were not significantly influenced by treatment.

\section{Primary and secondary outcomes in HCV-positive patients}

Normalization of $\gamma$ GT was observed among HCV-positive patients and was significantly greater in the RA vs the P group ( $54 \%$ vs $20 \%$, respectively, at $T_{12}, p=0.02 ; p<0.0001$ for baseline vs $T_{12}$ in the RA group). There were no significant differences in AST or ALT between $\mathrm{RA}$ and $\mathrm{P}$ in HCV-positive patients, either at baseline or at $T_{12}$. At $T_{12}$, mean blood glucose was $48 \%$ lower than at baseline in HCVpositive patients treated with RA, with no notable difference for $\mathrm{P}$ (not significant). In patients with a HOMA $>2.7$ (13 and 12 patients in RA and P groups, respectively), HOMA significantly decreased between baseline and $T_{12}$ with RA $(p<0.01)$ but not P. QoL (social activity and general health) improved between baseline and $T_{12}$ in HCVpositive patients treated with RA $(p<0.001)$.

Hyaluronic acid levels directly correlated with Ishak score $(p<0.01)$ and TNF- $\alpha$ levels with liver histology grading $(p<0.01)$; treatment did not influence either. Levels of MMP-2 and TGF- $\beta$ improved between baseline and $T_{12}$ in HCV-positive patients in the RA but not the P group (Fig. 4). No data are available for liver histology, because a second liver biopsy was available for only three patients.

Table 2

Statistical correlation at baseline between clinical and biochemical characteristics and liver histology parameters in the ITT population.

\begin{tabular}{|c|c|c|c|c|c|c|}
\hline Characteristic (r) & AST & ALT & BMI & Insulinemia & HOMA & TGF- $\beta$ \\
\hline Steatosis & - & $0.29(0.001)$ & - & - & - & - \\
\hline Lobular inflammation & $0.19(0.05)$ & - & - & - & - & - \\
\hline Ballooning & $0.17(0.05)$ & - & $0.27(0.002)$ & $0.19(0.03)$ & $0.22(0.01)$ & $-0.25(0.004)$ \\
\hline NAS & $0.43(0.001)$ & $0.24(0.02)$ & $0.33(0.001)$ & $0.22(0.03)$ & $0.25(0.01)$ & $-0.27(0.01)$ \\
\hline Portal infiltration & - & - & $0.29(0.003)$ & - & - & - \\
\hline Fibrosis & $0.22(0.01)$ & - & $0.18(0.04)$ & - & - & $-0.23(0.01)$ \\
\hline Siderosis & - & - & - & - & - & - \\
\hline Grading & - & - & - & - & $0.37(0.05)$ & - \\
\hline Ishak score & - & - & - & - & $0.41(0.02)$ & - \\
\hline
\end{tabular}

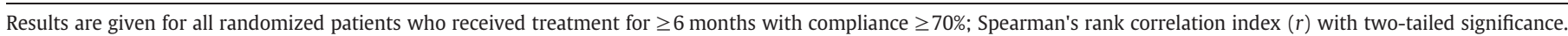

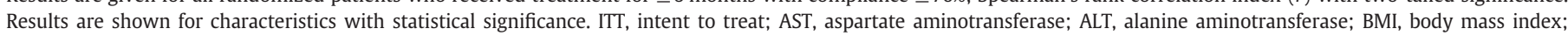
HOMA, homeostatic model assessment; TGF- $\beta$, transforming growth factor $\beta$; NAS, nonalcoholic fatty liver disease activity score. 


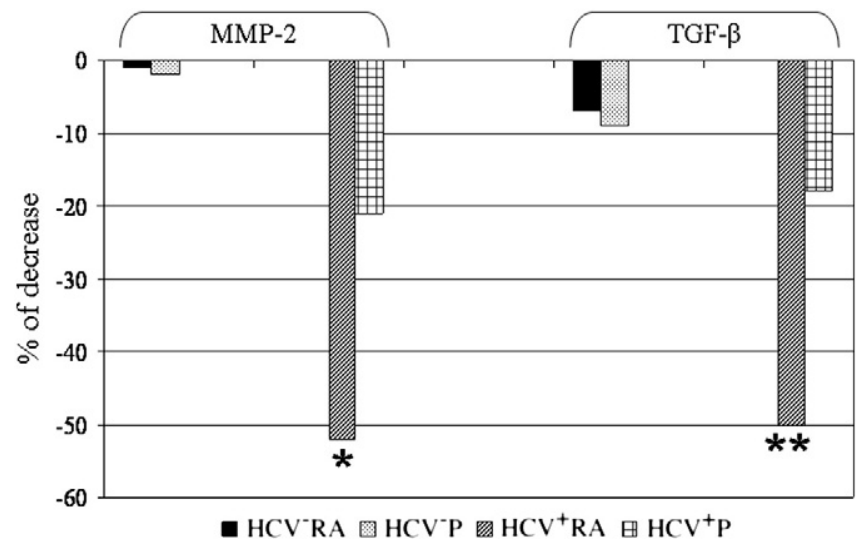

Fig. 4. Percentage of decrease at $T_{12}$ of some plasma markers of liver fibrogenesis (MMP-2 and TGF- $\beta$ ) in NAFLD and HCV-positive patients treated with RA or P. ${ }^{*} p=0.02$ and ${ }^{* *} p=0.004$ vs $T_{0}$.

\section{Additional analyses}

RA-treated NAFLD patients with histologic steatosis improvement showed a statistically significant ultrasonographic improvement ( $p<0.01$ vs baseline). In HCV-positive NAFLD patients, the ultrasonographic score improved in $100 \%$ of RA patients and $50 \%$ of $P$ patients ( $p=0.04$ between groups). In the latter group, MMP- 2 plasma values were significantly different at $T_{12}$ versus baseline $(p<0.01)$. In the NASH subgroup, with or without HCV infection, the proportion of patients with improved NAS was greater (mean 60\% versus 30\%) in patients with improved AST and $\gamma$ GT plasma levels, insulinemia, HOMA, and ultrasonographic steatosis score (not significant between groups).

\section{Discussion}

This is the first study to systematically assess the effects of silybin in NAFLD patients, with and without HCV infection. Patients treated for 12 months with RA, an oral complex containing silybin, phosphatidylcholine, and vitamin E, exhibited improvements in levels of transaminases and $\gamma \mathrm{GT}$, insulin resistance, and several aspects of liver histology. These improvements were similar to those noted in patients treated with pioglitazone and vitamin E [4,31]. However, in our study, body weight did not increase; conversely, we found an unexpected normalization of BMI in $15 \%$ of patients, which warrants further investigation.

Currently, no pharmacologic agents are approved for NAFLD or $\mathrm{NASH}$ treatment. Long-term treatment with insulin-sensitizing agents is debated [5], and chronic use of high-dose vitamin E may have negative effects [32]. Although the use of herbal products is increasing worldwide, their effects may not be exclusively beneficial [33]. Preliminary in vitro and in vivo studies of silybin showed an acceptable safety profile at doses exceeding $10 \mathrm{~g} /$ day [29,30,34,35]. This is important because NAFLD patients are generally younger than those with other hepatic conditions [36] and may require long-term treatment. Therefore, to confirm the preliminary positive data, we designed the present trial to investigate the use of silybin in a typical population of NAFLD patients.

Interestingly, among patients with chronic HCV infection, 12month treatment with RA improved $\gamma$ GT plasma levels, insulin resistance, plasma MMP-2 and TGF- $\beta$ levels (markers of fibrogenesis), and QoL. $\gamma$ GT is predictive of liver fibrosis and a marker of the metabolic syndrome; fibrogenesis, strongly affected by oxidative stress, is the predominant process of fibrosis; and elevated HOMA is a predictor of poor PEG-interferon/ribavirin treatment response and hepatocellular carcinoma in HCV-positive patients [37-41].
Our QoL findings echo recent observations by Seeff et al. [42], who reported benefits of silybin in patients with chronic HCV infection. Recently, attention has focused on silybin's effects on HCV replication [43-46]. In this study we found no notable changes in HCV RNA in patients treated with the stated dose of silybin (data not shown). Unfortunately we could not document possible effects of RA on liver histology in HCV-infected patients.

Silybin and silymarin have been employed as "hepatoprotectants" since the Greco-Roman era. In the United States and Europe approximately $65 \%$ of liver disease patients take herbal preparations, primarily those derived from the milk thistle plant (S. marianum) $[47,48]$. The absence of conclusive efficacy data may be due to the lack of large, well-designed trials. Studies have frequently been small, with a lack of appropriate randomization, insufficient information about the compound used, and wide variations in treatment duration. Additionally, in the case of milk thistle derivatives, silymarin, silybin, and other varying compounds are frequently used synonymously $[49,50]$.

We believe that our findings primarily relate to the antioxidant action of silybin. Silybin shows antioxidant activity both in vitro and in experimental animal models, inhibiting radical formation, binding some radical species (scavenger activity), interfering with lipid membrane peroxidation and permeability, and increasing the concentration of scavengers in a dose-dependent manner [51]. In human liver stellate cells, silybin inhibits extracellular signal-related kinase (ERK), MAP/ERK kinase (MEK), and Raf phosphorylation; reduces cell migration; and reduces TGF- $\beta$-induced synthesis of procollagen type I and secretion of MMP-2 [29]. ERK-, MEK-, and mitogenactivated protein kinase-related pathways are regulated and regulate Jun N-terminal kinase (JNK) expression, which has a pivotal role in obesity and insulin resistance [52]. Phosphatidylcholine, another component of the RA preparation, protects against oxidative stressmediated liver damage $[53,54]$. It is unlikely that the treatment effects are related to the vitamin E component, which is much lower (60 IU/ day) than doses with documented efficacy [4].

More recently, it has been confirmed, in a model of perfused rat liver, that the hepatoprotectant actions of silybin are related to decreased membrane lipid peroxidation, reduced free radical release, and restoration of the glutathione levels [55]. In vivo, in rats, silybin treatment, at doses similar to those used in humans, significantly protected against high-fat-induced fatty liver by stabilizing mitochondrial membrane fluidity, reducing serum content of alanine aminotransferase, decreasing hepatic malondialdehyde, and increasing superoxide dismutase and glutathione levels. Moreover, silybin enhanced the gene and protein expression of adiponectin and inhibited those of resistin. These results suggest that mitochondrial membrane stabilization, oxidative stress inhibition, and improved insulin resistance may be the essential mechanisms for the hepatoprotective effect of silybin on nonalcoholic fatty liver disease in rats [56].

In mice, silybin administration decreased HOMA-IR and serum ALT and markedly improved hepatic and myocardial damage. Silibinin reduced isoprostanes, 8-deoxyguanosine, and nitrites/nitrates in the liver and in the heart of $\mathrm{db} / \mathrm{db}$ mice fed the methionine-choline-deficient diet, whereas glutathione levels were restored to lean mouse levels in both tissues. Consistently, liver mitochondrial respiratory chain activity was significantly impaired in untreated mice and was completely restored in silibinin-treated animals. TNF- $\alpha$ was increased, whereas IL- 6 was decreased in both the liver and the heart of $\mathrm{db} / \mathrm{db}$ mice fed a methionine-choline-deficient diet. Silibinin reversed heart TNF- $\alpha$ and IL- 6 expression to control mouse levels. Indeed, liver JNK phosphorylation was reduced to control levels in treated animals [57]. In obese diabetic mice, silybin administration markedly decreased plasma levels of asymmetric dimethylarginine, an endogenous inhibitor of nitric oxide synthase that, as known, plays a pivotal role in endothelial dysfunction [58]. Finally, the antiviral action of silybin against HCV replication is also related to its antioxidant properties. In fact, silybin reduces the levels of some free 
radical species produced during HCV gene expression, and in this manner it interferes with the regulation of some proteins of the HCV core (for example, downregulation of HMOX-1 mRNA and upregulation of Nrf2 protein) necessary for HCV replication [59].

Although this trial was designed according to good clinical practice, several sources of bias are possible in this complex study designed 6 years ago. Its multicenter nature allowed the inclusion of more patients, but, despite standardized inclusion criteria, could have increased interpatient variability. Many patients did not agree to liver biopsy before/after treatment, which may relate to a lack of perceived disease among these predominantly asymptomatic patients. Researchers and patients may have doubted the benefits of a "dietary supplement," which could explain the challenges in recruiting patients and centers and the relatively high proportion of dropouts. We acknowledge that numerous endpoints were used and that conclusive therapeutic effects were not always demonstrated. The latter may relate to sample size; additional data obtained after this trial began suggested that the sample size assumptions were incorrect $[29,30]$.

In conclusion, we show that NAFLD patients treated for 12 months with RA exhibited improvements in transaminase levels, $\gamma$ GT levels, insulin resistance, and liver histology, without increases in body weight. These findings warrant further investigation, particularly for liver histology. However, this study provides valuable information that will facilitate the investigation of new therapeutic strategies for NAFLD patients with or without HCV infection.

\section{Acknowledgments}

We thank Gardiner-Caldwell Communications for general editing and styling assistance, which was funded by the Istituto Biochimico Italiano Lorenzini S.p.a, Italy. This study was funded by a grant from the Istituto Biochimico Italiano, Lorenzini S.p.a., Italy. The manuscript was written by the lead and corresponding author and was approved by all authors, who assume responsibility for the completeness of the data and the overall content of the article. This article was approved by the ethics committee of our university and includes data that have been presented at international meetings as abstract and poster/oral communications.

\section{Appendix A. Supplementary material}

Supplementary data to this article can be found online at doi:10. 1016/j.freeradbiomed.2012.02.008.

\section{References}

[1] Targher, G.; Day, C. P.; Bonora, E. Risk of cardiovascular disease in patients with non alcoholic fatty liver disease. N. Engl. J. Med. 363:1341-1350; 2010.

[2] Lok, A. S.; Everhart, J. E.; Chung, R. T.; Padmanabhan, L.; Greenson, J. K.; Shiffman, M. L.; Everson, G. T.; Lindsay, K. L.; Bonkovsky, H. L.; Di Bisceglie, A. M.; Lee, W. M.; Morgan, T. R.; Ghany, M. G.; Morishima, C., HALT-C Trial Group Hepatic steatosis in hepatitis C: comparison of diabetic and non-diabetic patients in the Hepatitis $C$ Antiviral Long-Term Treatment against Cirrhosis Trial. Clin. Gastroenterol. Hepatol. 5:245-254; 2007

[3] Loria, P.; Adinolfi, L. E.; Bellentani, S.; Bugianesi, E.; Grieco, A.; Fargion, S.; Gasbarrini, A.; Loguercio, C.; Lonardo, A.; Marchesini, G.; Marra, F.; Persico, M.; Prati, D.; Baroni, G. S. NAFLD Expert Committee of the Associazione Italiana per lo Studio del Fegato. Practice guidelines for the diagnosis and management of nonalcoholic fatty liver disease: a decalogue from the Italian Association for the Study of the Liver (AISF) Expert Committee. Dig. Liver Dis. 42:272-282; 2010.

[4] Sanyal, A. J.; Chalasani, N.; Kowdley, K. V.; McCullough, A.; Diehl, A. M.; Bass, N. M.; Neuschwander-Tetri, B. A.; Lavine, J. E.; Tonascia, J.; Unalp, A.; Van Natta, M.; Clark, J.; Brunt, E. M.; Kleiner, D. E.; Hoofnagle, J. H.; Robuck, P. R.; NASH CRN Pioglitazone, vitamin E, or placebo for nonalcoholic steatohepatitis. N. Engl. J. Med. 362:1675-1685; 2010.

[5] Musso, G.; Gambino, R.; Cassader, M.; Pagano, G. A meta-analysis of randomized trials for the treatment of nonalcoholic fatty liver disease. Hepatology 52:79-104; 2010.

[6] Gazak, R.; Walterova, D.; Kren, V. Silybin and silymarin-new and emerging applications in medicine. Curr. Med. Chem. 14:315-338; 2007.

[7] Tamayo, C.; Diamond, S. Review of clinical trials evaluating safety and efficacy of milk thistle (Sylibum marianum [L.] Gaertn.). Integr. Cancer Ther. 6:146-157; 2007.
[8] Amini, R.; Yazdanparast, R.; Aghazadeh, S.; Ghaffari, S. H. Anti-apoptotic and antiinflammatory effects of Silybum marianum in treatment of experimental steatohepatitis. Exp. Toxicol. Pathol. 63:569-574; 2011.

[9] Serviddio, G.; Bellanti, F.; Giudetti, A. M.; Gnoni, G. V.; Petrella, A.; Tamborra, R.; Romano, A. D.; Rollo, T.; Vendemiale, G.; Altomare, E. A silybin-phospholipids complex prevents mitochondrial dysfunction in a rodent model of nonalcoholic steatohepatitis. J. Pharmacol. Exp. Ther. 332:922-932; 2010.

[10] Di Sario, A.; Bendia, E.; Taffetani, S.; Omenetti, A.; Candelaresi, C.; Marzioni, M.; De Minicis, S.; Benedetti, A. Hepatoprotective and antifibrotic effect of a new silybin-phosphatidylcholine-vitamin E complex in rats. Dig. Liver Dis. 37:869-876; 2005.

[11] Maghrani, M.; Zeggwagh, N. A.; Lemhadri, A.; El Amraoui, M.; Michel, J. B.; Eddouks, M. Study of the hypoglycaemic activity of Fraxinus excelsior and Silybum marianum in an animal model of type 1 diabetes mellitus. J. Ethnopharmacol. 91:309-316; 2004.

[12] Lirussi, F.; Beccarello, A.; Zanette, G.; De Monte, A.; Donadon, V.; Velussi, M.; Crepaldi, G. Silybin- $\beta$-cyclodextrin in the treatment of patients with diabetes mellitus and alcoholic liver disease: efficacy study of a new preparation of an anti-oxidant agent. Diabetes Nutr. Metab. 15:222-231; 2002.

[13] Huseini, H. F.; Larijani, B.; Heshmat, R.; Fakhrzadeh, H.; Radjabipour, B.; Toliat, T.; Raza, M. The efficacy of Silybum marianum (L.) Gaertn. (silymarin) in the treatment of type II diabetes: a randomized, double-blind, placebo-controlled, clinical trial. Phytother. Res. 20:1036-1039; 2006.

[14] Wen, Z.; Dumas, T. E.; Schrieber, S. J.; Hawke, R. L.; Fried, M. W.; Smith, P. C. Pharmacokinetics and metabolic profile of free, conjugated, and total silymarin flavonolignans in human plasma after oral administration of milk thistle extract. Drug Metab. Dispos. 36:65-72; 2008.

[15] Yanyu, X.; Yunmei, S.; Zhipeng, C.; Qineng, P. The preparation of silybinphospholipid complex and the study on its pharmacokinetics in rats. Int. J. Pharm. 307:77-82; 2006.

[16] Jia, L. J.; Zhang, D. R.; Li, Z. Y.; Feng, F. F.; Wang, Y. C.; Dai, W. T.; Duan, C. X.; Zhang, Q. Preparation and characterization of silybin-loaded nanostructured lipid carriers. Drug Deliv. 2009 Nov 26. [Epub ahead of print].

[17] Flaig, T. W.; Gustafson, D. L.; Su, L. J.; Zirrolli, J. A.; Crighton, F.; Harrison, G. S.; Pierson, A. S.; Agarwal, R.; Glodé, L. M. A phase I and pharmacokinetic study of silybin-phytosome in prostate cancer patients. Invest. New Drugs 25:139-146; 2007.

[18] Kidd, P. M. Bioavailability and activity of phytosome complexes from botanical polyphenols: the silymarin, curcumin, green tea, and grape seed extracts. Altern. Med. Rev. 14:226-246; 2009.

[19] Loguercio, C.; Di Pierro, M.; Di Marino, M. P.; Federico, A.; Disalvo, D.; Crafa, E.; Tuccillo, C.; Baldi, F.; Del Vecchio Blanco, C. Drinking habits of subjects with HCV-related chronic liver disease: the prevalence and effect on clinical, virological and pathological aspects. Alcohol Alcohol. 35:296-301; 2000.

[20] Conigrave, K. M.; Hall, W. D.; Saunders, J. B. The AUDIT questionnaire: choosing a cutoff score. Alcohol Use Disorder Identification Test. Addiction 90:1349-1356 1995.

[21] Kleiner, D. E.; Brunt, E. M.; Van Natta, M.; Behling, C.; Contos, M. J.; Cummings, O. W.; Ferrell, L. D.; Liu, Y. C.; Torbenson, M. S.; Unalp-Arida, A.; Yeh, M.; McCullough, A. J.; Sanyal, A. J. Design and validation of a histological scoring system for nonalcoholic fatty liver disease. Hepatology 41:1313-1321; 2005.

[22] Lee, S. S.; Park, S. H.; Kim, H. J.; Kim, S. Y.; Kim, M. Y.; Kim, D. Y.; Suh, D. J.; Kim, K. M.; Bae, M. H.; Lee, J. Y.; Lee, S. G.; Yu, E. S. Non-invasive assessment of hepatic steatosis: prospective comparison of the accuracy of imaging examinations. J. Hepatol. 52:570-585; 2010.

[23] David, K.; Kowdley, K. V.; Unalp, A.; Kanwal, F.; Brunt, E. M.; Schwimmer, J. B., NASH CRN Research Group Quality of life in adults with nonalcoholic fatty liver disease: baseline data from the nonalcoholic steatohepatitis clinical research network. Hepatology 49:1904-1912; 2009.

[24] Schaan, B. D.; Portal, V. L.; de Ugarte, M. T.; Dias, A. A.; Hatem, D. M. Emerging risk factors and early atherosclerosis indices in subjects with impaired glucose tolerance. Diabetes Metab. 31:581-587; 2005.

[25] Tilg, H.; Diehl, A. M. Cytokines in alcoholic and nonalcoholic steatohepatitis N. Engl. J. Med. 343:1467-1476; 2000.

[26] Manning, D. S.; Afdhal, N. H. Diagnosis and quantification of fibrosis. Gastroenterology 134:1670-1681; 2008.

[27] Brunt, E. M. Nonalcoholic steatohepatitis. Semin. Liver Dis. 24:3-20; 2004.

[28] Ishak, K.; Baptista, A.; Bianchi, L.; Callea, F.; De Groote, J.; Gudat, F.; Denk, H.; Desmet, V.; Korb, G.; MacSween, R. N. Histological grading and staging of chronic hepatitis. J. Hepatol. 22:696-699; 1995.

[29] Federico, A.; Trappoliere, M. Tuccillo, C; de Sio, I. Di Leva, A. Del Vecchio Blanco, C.; Loguercio, C. A new silybin-vitamin E-phospholipid complex improves insulin resistance and liver damage in patients with non-alcoholic fatty liver disease: preliminary observations. Gut 55:901-902; 2006.

[30] Loguercio, C.; Federico, A.; Trappoliere, M.; Tuccillo, C.; de Sio, I.; Di Leva, A.; Niosi, M.; D'Auria, M. V.; Capasso, R.; Del Vecchio Blanco, C., Real Sud Group The effect of a silybin-vitamin E-phospholipid complex on nonalcoholic fatty liver disease: a pilot study. Dig. Dis. Sci. 52:2387-2395; 2007.

[31] Vuppalanchi, R.; Chalasani, N. Nonalcoholic fatty liver disease and nonalcoholic steatohepatitis: selected practical issues in their evaluation and management. Hepatology 49:306-317; 2009.

[32] Miller III, E. R.; Pastor-Barriuso, R.; Dalal, D.; Riemersma, R. A.; Appel, L. J.; Guallar E. Meta-analysis: high-dosage vitamin E supplementation may increase all-cause mortality. Ann. Intern. Med. 142:37-46; 2005.

[33] Seeff, L. B. Are herbals as safe as their advocates believe? J. Hepatol. 50:13-16; 2009. 
[34] Trappoliere, M.; Caligiuri, A.; Schmid, M.; Bertolani, C.; Failli, P.; Vizzutti, F.; Novo, E.; di Manzano, C.; Marra, F.; Loguercio, C.; Pinzani, M. Silybin, a component of sylimarin, exerts anti-inflammatory and anti-fibrogenic effects on human hepatic stellate cells. J. Hepatol. 50:1102-1111; 2009.

[35] Wu, J. W.; Lin, L. C.; Tsai, T. H. Drug-drug interactions of silymarin on the perspective of pharmacokinetics. J. Ethnopharmacol. 12:185-193; 2009.

[36] Loguercio, C.; De Simone, T.; D'Auria, M. V.; de Sio, I.; Federico, A.; Tuccillo, C.; Abbatecola, A. M.; Del Vecchio Blanco, C., Italian AISF Clinical Group Non-alcoholic fatty liver disease: a multicentre clinical study by the Italian Association for the Study of the Liver. Dig. Liver Dis. 36:398-405; 2004.

[37] Devers, M. C.; Campbell, S.; Shaw, J.; Zimmet, P.; Simmons, D. Should liver function tests be included in definitions of metabolic syndrome? Evidence from the association between liver function tests, components of metabolic syndrome and prevalent cardiovascular disease. Diabet. Med. 25:523-529; 2008.

[38] Castera, L.; Pinzani, M. Biopsy and non-invasive methods for the diagnosis of liver fibrosis: does it take two to tango? Gut 59:861-866; 2010.

[39] Popov, Y.; Schuppan, D. Targeting liver fibrosis: strategies for development and validation of antifibrotic therapies. Hepatology 50:1294-1306; 2009.

[40] Tiniakos, D. G.; Vos, M. B.; Brunt, E. M. Nonalcoholic fatty liver disease: pathology and pathogenesis. Annu. Rev. Pathol. 5:145-171; 2010.

[41] Hung, C. H.; Wang, J. H.; Hu, T. H.; Chen, C. H.; Chang, K. C.; Yen, Y. H.; Kuo, Y. H.; Tsai, M. C.; Lu, S. N.; Lee, C. M. Insulin resistance is associated with hepatocellular carcinoma in chronic hepatitis C infection. World J. Gastroenterol. 16:2265-2271; 2010.

[42] Seeff, L. B.; Curto, T. M.; Szabo, G.; Everson, G. T.; Bonkovsky, H. L.; Dienstag, J. L.; Shiffman, M. L.; Lindsay, K. L.; Lok, A. S.; Di Bisceglie, A. M.; Lee, W. M.; Ghany, M. G., HALT-C Trial Group Herbal product use by persons enrolled in the Hepatitis C Antiviral Long-Term Treatment against Cirrhosis (HALT-C) Trial. Hepatology 47: 605-612; 2008.

[43] Polyak, S. J.: Morishima, C.: Shuhart, M. C.: Wang C. C.: Liu, Y : Lee, D. Y. Inhibition of T-cell inflammatory cytokines, hepatocyte NF- $\kappa$ B signaling, and HCV infection by standardized silymarin. Gastroenterology 132:1925-1936; 2007.

[44] Ferenci, P.; Scherzer, T. M.; Kerschner, H.; Rutter, K.; Beinhardt, S.; Hofer, H.; Schöniger-Hekele, M.; Holzmann, H.; Steindl-Munda, P. Silibinin is a potent antiviral agent in patients with chronic hepatitis $C$ not responding to pegylated interferon/ribavirin therapy. Gastroenterology 135:1561-1567; 2008.

[45] Biermer, M.; Berg, T. Rapid suppression of hepatitis C viremia induced by intravenous silibinin plus ribavirin. Gastroenterology 137:390-391; 2009.

[46] Ahmed-Belkacem, A.; Ahnou, N.; Barbotte, L.; Wychowski, C.; Pallier, C.; Brillet R.; Pohl, R. T.; Pawlotsky, J. M. Silibinin and related compounds are direct inhibitors of hepatitis C virus RNA-dependent RNA polymerase. Gastroenterology 138 : 1112-1122; 2010.
[47] Stickel, F.; Shuppan, D. Herbal medicine in the treatment of liver diseases. Dig. Liver Dis. 39:293-304; 2007.

[48] Jacobs, B. P.; Dennehy, C.; Ramirez, G.; Sapp, J.; Lawrence, V. A. Milk thistle for the treatment of liver disease: a systematic review and meta-analysis. Am. J. Med. 113:506-515; 2002.

[49] Rambaldi, A.; Jacobs, B. P.; Iaquinto, G.; Gluud, C. Milk thistle for alcoholic and/or hepatitis B or C liver diseases-a systematic Cochrane Hepato-Biliary Group review with meta-analyses of randomized clinical trials. Am. J. Gastroenterol. 100: 2583-2591; 2005

[50] Simánek, V.; Kren, V.; Ulrichová, J.; Vicar, J.; Cvak, L. Silymarin: what is in the name ...? An appeal for a change of editorial policy. Hepatology 32:442-444; 2000.

[51] Loguercio, C.; Festi, D. Silybin and the liver: from basic research to clinical practice. World J. Gastroenterol. 17:2288-2301; 2011.

[52] Aghazadeh, S.; Yazdanparast, R. Inhibition of JNK along with activation of ERK1/2 MAPK pathways improve steatohepatitis among the rats. Clin. Nutr. 29:381-385; 2010.

[53] Ikura, Y.; Ohsawa, M.; Suekane, T.; Fukushima, H.; Itabe, H.; Jomura, H.; Nishiguchi, S.; Inoue, T.; Naruko, T.; Ehara, S.; Kawada, N.; Arakawa, T.; Ueda, M. Localization of oxidized phosphatidylcholine in nonalcoholic fatty liver disease: impact on disease progression. Hepatology 43:506-514; 2006.

[54] Kharbanda, K. K. Mailliard, M. E. Baldwin, C. R. Beckenhauer, H. C. Sorrell, M. F. Tuma, D. J. Betaine attenuates alcoholic steatosis by restoring phosphatidylcholine generation via the phosphatidylethanolamine methyltransferase pathway. J. Hepatol. 46:314-321; 2007.

[55] Colturato, C. P.; Constantin, R. P.; Maeda Jr., A. S.; Constantin, R. P.; Yamamoto, N. S.; Bracht, A.; Ishii-Iwamoto, E. L.; Constantin, J. Metabolic effects of silibinin in the rat liver. Chem. Biol. Interact. 195:119-132; 2012.

[56] Yao, J.; Zhi, M.; Minhu, C. Effect of silybin on high-fat-induced fatty liver in rats. Braz. J. Med. Biol. Res. 44:652-659; 2011.

[57] Salamone, F.; Galvano, F.; Marino, A.; Paternostro, C.; Tibullo, D.; Bucchieri, F.; Mangiameli, A.; Parola, M.; Bugianesi, E.; Li Volti, G. Silibinin improves hepatic and myocardial injury in mice with nonalcoholic steatohepatitis. Dig. Liver Dis. 2011 Dec 23. [Epub ahead of print].

[58] Li Volti, G.; Salomone, S.; Sorrenti, V.; Mangiameli, A.; Urso, V.; Siarkos, I.; Galvano, F.; Salamone, F. Effect of silibinin on endothelial dysfunction and ADMA levels in obese diabetic mice. Cardiovasc. Diabetol. 10:62; 2011.

[59] Mehrab-Mohseni, M.; Sendi, H.; Steuerwald, N.; Ghosh, S.; Schrum, L. W.; Bonkovsky, H. L. Legalon-SIL downregulates HCV core and NS5A in human hepatocytes expressing full-length HCV. World J. Gastroenterol. 17:1694-1700; 2011. 